\title{
Effects of Trunk Postures and Shoulder Flexion Angles on the Muscular Activity of Scapular Upward Rotators
}

\author{
Jong-Rak Park ${ }^{1}$, Jong-Hyuck Weon ${ }^{2, *}$ \\ ${ }^{1}$ Department of Rehabilitation Science, Grauate School, Daegu University, South Korea \\ ${ }^{2}$ Department of Physical Therapy, Joongbu University, South Korea
}

Received April 6, 2021; Revised May 28, 2021; Accepted June 20, 2021

\begin{abstract}
Cite This Paper in the following Citation Styles
(a): [1] Jong-Rak Park, Jong-Hyuck Weon, "Effects of Trunk Postures and Shoulder Flexion Angles on the Muscular Activity of Scapular Upward Rotators, "International Journal of Human Movement and Sports Sciences, Vol. 9, No. 5, pp. 858 - 862, 2021. DOI: 10.13189/saj.2021.090505.
\end{abstract}

(b): Jong-Rak Park, Jong-Hyuck Weon (2021). Effects of Trunk Postures and Shoulder Flexion Angles on the Muscular Activity of Scapular Upward Rotators. International Journal of Human Movement and Sports Sciences, 9(5), 858 - 862. DOI: $10.13189 /$ saj.2021.090505.

Copyright $\odot 2021$ by authors, all rights reserved. Authors agree that this article remains permanently open access under the terms of the Creative Commons Attribution License 4.0 International License

\begin{abstract}
The forward bending posture is a risk factor that can cause musculoskeletal injury for workers working in industry. Previous studies have been largely concerned with examining the effects of turtle neck, slouched posture, and kyphosis on the scapular upward rotators. However, no studies have investigated the effect of forward bending of the trunk on the scapular upward rotators to date. The purpose of this study was to investigate the effect of forward tilting of the trunk and the shoulder flexion angle on the muscular activity of the Upper trapezius. The participant population comprised of 10 male adults and 20 shoulders in total were assessed. To begin with, EMG electrodes were attached to the upper trapezius muscles. The participants were instructed to perform isometric shoulder flexion at $120^{\circ}$ angles in upright standing and forward bending postures. The muscle activity was measured by surface electromyography to obtain a root mean square value and was expressed as a percentage of the mean maximal voluntary isometric contraction (\%MVIC). A paired t-test was used to determine the changes in muscle activity according to trunk posture and a shoulder flexion angle. A paired t-test was performed at $\alpha=.05$. The result of Significant main effect for trunk posture was found for the upper trapezius muscles $(p<.05)$. Additionally, a significant main effect for shoulder flexion angle was revealed in the upper trapezius muscles $(\mathrm{p}<.05)$. The results demonstrate that forward bending of the trunk may increase the activity of the upper trapezius. Therefore, industrial workers who manifest hyperactivation of the
\end{abstract}

upper trapezius should maintain upright trunk posture while working.

Keywords Forward Bending, Forward Head Posture, Scapular Upward Rotators, Upper Trapezius

\section{Introduction}

Forward bending posture (FBP) is a risk factor that can cause musculoskeletal pain in industrial workers and is particularly known to have a close association with lumber pain [1-3]. For this reason, studies related to FBP and lumber pain have continued to be conducted [4-6]. However, improper trunk postures can affect muscles in not only the lower back, but also the neck and shoulders [7-9]. Slouched postures are also reported to have influenced the alignment of the neck and scapula, thereby influencing movements or muscle activities of the scapula and upper arms [10-11].

Mechanical cervical dysfunction is a common disorder in other populations, resulting a pain and limited range of motion (ROM) negatively affect everyday life, activities and quality of life [12]. Poor spinal postures that can affect scapular alignment, movements, muscle activity include forward head posture (FHP) [13] and excessive thoracic kyphosis in the back [10-11], [14]. According to previous studies, FHP reduced the serratus anterior muscle 
activity compared to normal postures and increased the upper trapezius muscle activity [14-15]. In addition, slouched postures also caused statistically significant changes in scapular movement and muscle activity of the scapular upward rotator [11], [16].

Researchers compared scapular movements and muscle activities between individuals with FHP and individuals with normal postures and found that the FHP group had greater anterior tilting of the scapula than the normal posture group at a statistically significant level [14]. They attributed this difference to more serious kyphosis in the spine [10], [17] and shortening of the pectoralis minor muscle [17]. In addition, some studies argued that kyphosis becomes a factor that reduces scapular upward rotation [10-11].

The FBP changes the direction of gravity applied to the body. Moreover, the direction of gravity applied to each joint or muscle of the body changes interactions between agonists and antagonists, as well as influences the activity of each muscle [18]. For this reason, in the case of performing an FBP and lifting arms, even those with normal alignment of the trunk will show similar muscle activity changes to those with slouched postures. However, no research has investigated changes in scapular movement or muscle activity when individuals with normal cervical and scapular alignments move arms in an FBP.

Therefore, the present study aimed to identify changes in muscle activity of the scapular upward rotator when individuals with normal cervical and trunk alignments lifted arms in an FBP.

The study's hypotheses are as follows: when the subjects lift their arms at a $120^{\circ}$ angle in each of an upright standing position (USP) and a $30^{\circ} \mathrm{FBP}$, their upper trapezius muscle activities will show a statistically significant difference.

\section{Methods}

\subsection{Participants}

In this study, the experimental group was only male subject due to difficulties in the measurement site or measurement posture. G-power analysis software was used with G*power software ver. 3.1.9.7 (Franz Faul, University of Kiel, Kiel, Germany). The sample size was calculated with 0.90 power, 0.05 alpha level, and 0.8 effect size. This result indicated that a necessary sample size was at least 19 subjects for this study. In the present study, 20 subjects were included considering the dropouts. In total, 10 adult men participated in this study and an experiment was conducted on 20 scapulae that met the selection criteria of the study. All subjects were provided with a full explanation of the purpose and methods prior to the study, and spontaneously participated after signing the informed consent. The selection criteria for subjects were as follows:

(1) Those who have no limitations in the active range of motion of the shoulder joint.

(2) Those who have neither pain nor discomfort that could affect their shoulder joint movements.

(3) Those who have no history of orthopedic surgery or legions in the shoulder joint.

The subjects were tested in random. In total, 10 subjects participated in the study and 20 shoulder joints were examined through the experiment. General characteristics of the subjects are shown in Table 1.

Table 1. General characteristics of the subjects

\begin{tabular}{ccc}
\hline & Mean \pm SD & Range \\
\hline Age $($ year $)$ & $24.90 \pm 3.73$ & $20 \sim 33$ \\
Height $(\mathrm{cm})$ & $176.90 \pm 5.47$ & $166 \sim 183$ \\
Weight $(\mathrm{kg})$ & $74.20 \pm 9.37$ & $61 \sim 90$ \\
\hline
\end{tabular}

Mean \pm standard deviation

\subsection{Procedures}

The surface electromyography (SEMG) system Noraxon TeleMyo 2400 (Noraxon Inc, Scottsdale, AZ, USA) was used to measure the upper trapezius muscle activity when the subjects lifted arms to a $120^{\circ}$ angle in two trunk postures. The Noraxon MyoResearch XP 1.06 software was employed to collect and analyze the muscle activity of scapular upward rotators. The sample rating of EMG signals was set at $1,000 \mathrm{~Hz}$ and the band pass filter was set at 10 to $450 \mathrm{~Hz}$ to remove electrocardiogram signals that are nosy biosignals [19].

The areas to which EMG electrodes would be attached were shaved to measure the upper trapezius muscle activity, and the electrodes were attached with the electrode spacing of $2 \mathrm{~cm}$ after the respective areas of skin were wiped with alcohol swabs to reduce skin resistance. The electrodes for the upper trapezius were placed parallel to the direction of muscle fibers in the midpoint between the spinous process of the seventh cervical vertebra and the posterolateral acromion of the scapula [20].

While the subjects were maintaining $120^{\circ}$ shoulder flexion in a USP and $30^{\circ} \mathrm{FBP}$, their upper trapezius muscle activities were measured.

Each subject performed each of the USP and FBP while holding a $1.5 \mathrm{~kg}$ dumbbell in their hand, lifted their arm to contact a target-bar that had been preinstalled at a height that makes $120^{\circ}$ shoulder flexion, maintained isometric contraction for about five seconds, and then lowered the arm. They performed this motion three times each session and six times in total. Here, the movement of the arm was based on the sagittal plane, and one-minute break was given between each measurement to minimize the impact of muscular fatigue on the experimental results (Figure 1).

The experiment was conducted by two testers who had 
a license to practice physical therapy and at least four years of clinical experience. Once tester operated computers to run EMG program and the other tester controlled the experimental procedures including testing postures and methods. Both testers fully understood the entire experimental process and both the testers and subjects were blinded to the implications of the study's purpose and results.

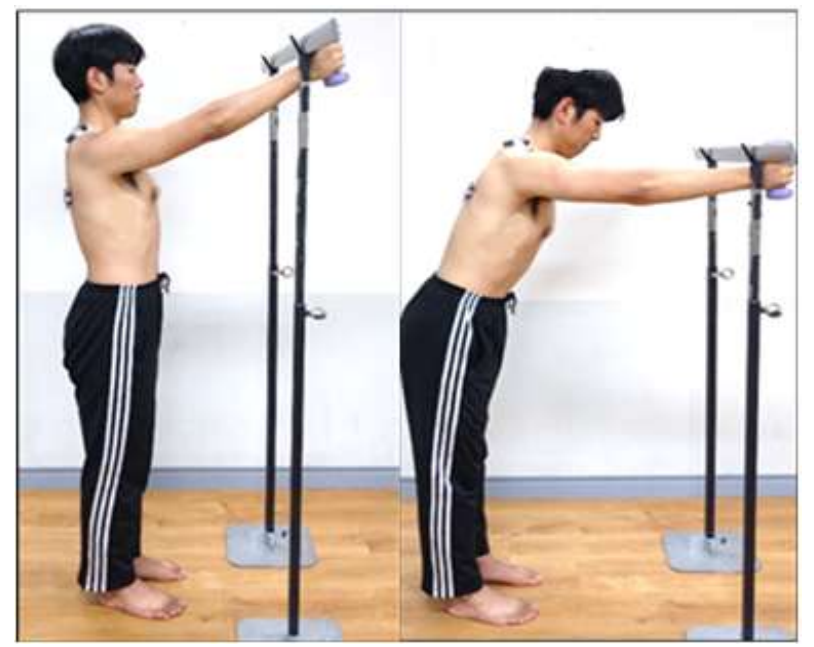

Figure 1. Two positions for measuring the upper trapezius muscle activity: upright standing with $120^{\circ}$ shoulder flexion and $30^{\circ}$ trunk forward bending with $120^{\circ}$ shoulder flexion.

\subsection{Statistical Analysis}

The EMG signals of the upper trapezius collected for five seconds in each posture were processed using the root mean square (RMS) method, and then the mean of the values for three seconds excluding the first and last seconds was adopted for data analysis. According to the muscle strength assessment method by Researchers [21], the muscle activity during maximal voluntary isometric contraction (MVIC) was measured. Based on the resulting values, the \% MVIC values were obtained and used.

The data collected through the experiment were analyzed using the statistical software SPSS Statistics 21 Window. A paired sample t-test was employed to examine changes in muscle activity depending on the angle of shoulder flexion. The statistical significance level was set at .05 .

\section{Results}

Upper trapezius muscle activities were conducted according to trunk postures and shoulder flexion angles.

Table 2 presents the upper trapezius muscle activities measured while the subjects were maintaining $120^{\circ}$ shoulder flexion in two positions of USP and FBP. According to the analysis results, during $120^{\circ}$ shoulder flexion in the USP and FBP, the upper trapezius muscle activities showed statistically significant differences between the positions $(\mathrm{p}<.05)$.

Table 2. Muscle activities in $120^{\circ}$ shoulder flexion for two trunk postures

\begin{tabular}{|c|c|c|c|c|c|}
\hline & & \multicolumn{3}{|c|}{ Descriptive Statistics } & \multirow[b]{2}{*}{$t(p)$} \\
\hline \multicolumn{2}{|c|}{ Category } & $\mathrm{N}$ & Mean & Standard & \\
\hline \multirow{2}{*}{$\begin{array}{l}\text { Upper } \\
\text { trapezius } \\
\text { muscle } \\
\text { activity }\end{array}$} & $\begin{array}{l}\text { USP } \\
120^{\circ}\end{array}$ & 20 & 40.73 & 20.46 & \multirow{2}{*}{$-4.851(.000)^{* * *}$} \\
\hline & $\begin{array}{l}\text { FBP } \\
120^{\circ}\end{array}$ & 20 & 51.67 & 21.90 & \\
\hline
\end{tabular}

USP: uplight standing posture

FBP: forward banding posture

\section{Discussion}

This study was conducted to understand changes in upper trapezius muscle activity according to the angles of a trunk forward bending and shoulder flexion.

Based on the study results, an increase in shoulder flexion angle resulted in a corresponding increase in upper trapezius muscle activity, which was statistically significant $(p<.05)$. The result is a similar to previous studies in which the muscle activity of scapular upward rotators was measured while the subjects were lifting their arms [22-24], and can also be explained with the length-tension relationship [25-26]. Specifically, lifting the arm at a larger angle may have led the muscle to maintain a more shortened length, and as a result, increased the muscle activity.

The upper trapezius muscle activity is known to be affected by various factors. The muscle activity changes according to head positions such as FHP [13], [15] and is also influenced by excessive thoracic kyphosis [10] and visual feedback [27]. In addition, some previous studies reported that the upper trapezius muscle activity showed differences depending on the weight applied to the hand or grip strength and the flexion angle of the glenohumeral joint [13], [28-30].

According to a comprehensive review of the above studies, in the case of having a FHP or thoracic kyphosis, the subjects showed a general pattern of increases in upper trapezius muscle activity and reductions in serratus anterior and lower trapezius muscle activities. These changes occasionally become a cause of the shoulder impingement syndrome by reducing scapular upward rotation and increasing the scapular anterior tilt [13], [18], [26].

Unlike conventional studies on the same topic, this study involved normal adults without any FHP or thoracic kyphosis, and controlled the experimental process not to cause FHP or kyphosis in the subjects and instructed them to perform the FBP by bending only the hip joint. For this 
reason, it is problematic to explain results of the present study in comparison with those of the previous studies. A primary factor that changed the upper trapezius in the present study can be explained as changes in the direction of gravity due to the FBP. In the USP that is perpendicular to the force of gravity, gravity works as an external force causing scapular depression and downward rotation. However, as observed in this study, gravity during the FBP acts as an external force causing not only scapular depression and downward rotation, but also abduction [31].

As this shows, while the arm is being lifted, scapular upward rotators are contracted against gravity. In the USP, muscle activities for scapular elevation and upward rotation are activated. However, in the FBP, gravity also becomes an external force leading to abduction. Therefore, muscle activities are required not only for scapular elevation and upward rotation, but also for scapular adduction. Hence, scapular elevators and upward rotators as well as the upper trapezius that works as a scapular adductor may have been further activated.

There are some limitations in generalizing the findings of this study given that it involved only normal adult men as subjects. Therefore, future studies are required to investigate changes in the muscle activity of scapular upward rotators in industrial workers at various trunk inclination angles.

\section{Conclusions}

The present study was conducted to investigate the effects of FBP and shoulder flexion angles on the upper trapezius muscle activity. Ten normal adult men participated in the study and 20 scapulae were tested. The subjects were instructed to perform two positions of lifting the shoulder joint at a $120^{\circ}$ angle in each of the USP and $30^{\circ} \mathrm{FBP}$ while holding a $1.5 \mathrm{~kg}$ dumbbell. During each position, their EMG signals of the upper trapezius were measured using an EMG device. Every muscle activity measured using SEMG was processed to have the root mean square value, and the activity of each muscle was calculated as the \%MVIC value of the muscle activity during maximum voluntary isometric contraction for analysis. To examine changes in the upper trapezius muscle activity according to shoulder flexion angles in the USP and FBP, the statistical significance of the resulting data for each condition was tested using a paired t-test. The statistical significance level was set at .05 .

The findings of this study suggest that the FBP and shoulder flexion angles can cause changes of increasing the upper trapezius muscle activity. Therefore, it is recommended that workers who experience excessive activation of the upper trapezius or frequently use their arms should use their arms while maintaining a USP during work.

\section{REFERENCES}

[1] W. E. Hoogendoorn, P. M. Bongers, H. C. de Vet, et al. Flexion and rotation of the trunk and lifting at work are risk factors for low back pain: results of a prospective cohort study. Spine. 25:3087-3092, 2000.

[2] M. S. Kerr, J.W. Frank, Shannon HS, et al. Biomechanical and psychosocial risk factors for low back pain at work. Am J Public Health. 91:1069-1075, 2001.

[3] E. Vingard, L. Alfredsson, M. Hagberg, et al. To what extent do current and past physical and psychosocial occupational factors explain care-seeking for low back pain in a working population? Results from the Musculoskeletal Intervention Center-Norrtalje Study. Spine. 25:493-500, 2000 .

[4] L. K. Lunde, M.Koch, S. L. Merkus, et al. Associations of objectively measured forward bending at work with low-back pain intensity: a 2-year follow-up of construction and healthcare workers. Occup Environ Med.76(9):660-667, 2019.

[5] M. Osumi, M. Sumitani, Y. Otake, et al. Kinesiophobia modulates lumbar movements in people with chronic low back pain: a kinematic analysis of lumbar bending and returning movement. Eur Spine J. 28(7):1572-1578, 2019.

[6] I. Shojaei, C. Suri, B. Bazrgari. The effects of backpack type on lumbo-pelvic coordination during trunk bending and return tasks. Ergonomics. 61(10):1355-1363, 2018.

[7] S. S. Lynch, C. A. Thigpen, J. P. Mihalik, et al. The effects of an exercise intervention on forward head and rounded shoulder postures in elite swimmers. Br J Sports Med. 44(5):376-381, 2010.

[8] G. P. Szeto, L. Straker, S. Raine. A field comparison of neck and shoulder postures in symptomatic and asymptomatic office workers. Appl Ergon. 33:75-84, 2002

[9] W. Lim. Tensile Force Transmission from the Upper Trunk to the Contralateral Lower Leg throughout the Posterior Oblique Sling System. International Journal of Human Movement and Sports Sciences, 9(2), 294-300, 2021. DOI: 10.13189/saj.2021.090217.

[10] M. A. Finley, R. Y. Lee. Effect of sitting posture on 3-dimensional scapular kinematics measured by skin-mounted electromagnetic tracking sensors. Arch Phys Med Rehabil. 84(4):563-568, 2003.

[11] M. Kebaetse, P. Mcclure, N. A. Pratt. Thoracic position effect on shoulder range of motion, strength, and three-dimensional scapular kinematics. Arch Phys Med Rehabil. 80:945-950, 1999.

[12] E.Mohamed, R. Elrazik. Sustained Natural Apophyseal Glides versus Positional Release Therapy in the Treatment of Chronic Mechanical Neck Dysfunction. International Journal of Human Movement and Sports Sciences, 8(6), 384-394, 2020. DOI: 10.13189/saj.2020.080610.

[13] P. M. Ludewig, T. M. Cook, D. A. Nawoczenski. Three-dimensional scapular orientation and muscle activity 
at selected positions of humeral elevation. J Orthop Sports Phys Ther. 24(2):57-65, 1996.

[14] C. A. Thigpen, D. A. Padua, L. A. Michener, et al. Head and shoulder posture affect scapular mechanics and muscle activity in overhead tasks. $\mathrm{J}$ Electromyogr Kinesiol. 20(4):701-709, 2010.

[15] J. H. Weon, J. S.Oh, H. S Cynn, et al. Influence of forward head posture on scapular upward rotators during isometric shoulder flexion. J Bodyw Mov Ther. 14(4):367-374, 2010.

[16] E. M. Malmström, J.Olsson, J. Baldetorp, et al. Aslouched body posture decreases arm mobility and changes muscle recruitment in the neck and shoulder region. Eur J Appl Physiol. 115(12):2491-2503, 2015.

[17] J. D. Borstad, P. M. Ludewig. The effect of long versus short pectoralis minor resting length on scapular kinematics in healthy individuals. J Orthop Sports Phys Ther. 35:227-238, 2005.

[18] S. A. Sahrmann. Diagnosis and Treatment of Movement Impairment Syndrome. St Louis: Mosby, pp 41-44, 2002.

[19] G.Lu, J. S.Brittain, P. Holland, et al. Removing ECG noise from surface EMG signals using adaptive filtering. Neurosci Lett. 462(1):14-19, 2009.

[20] E. Criswell. Cram's introduction to surface electromyography. Jones and Bartlett Publishers, 2010.

[21] F. P. Kendall, E. K. McCreary, P. Provance. Muscles: Testing and function with posture and pain. 5th ed, Baltimore, MD: Lippincott Williams \& Wilkins, 2005.

[22] W. J. Choi, H. S. Cynn, C. H. Lee, et al. Shrug exercises combined with shoulder abduction improve scapular upward rotator activity and scapular alignment in subjects with scapular downward rotation impairment. J Electromyogr Kinesiol. 25(2):363-370, 2015.
[23] N. Y. Jeon, J. H. Weon, K. H. Lee, et al. Effect of scapular downward rotator stretch exercises on scapular upward rotator activity during arm elevation in subjects with scapular downward rotation syndrome. Indi JPhysiothr Occu Ther. 13(2):53-58, 2019.

[24] M. H. Kang, J. Y. Choi, J. S Oh. Effects of crossed brassiere straps on pain, range of motion, and electromyographic activity of scapular upward rotators in women with scapular downward rotation syndrome. PM R.7(12):1261-1268, 2015.

[25] L. De Wilde, E. Audenaert, E. Barbaix, et al. Consequences of deltoid muscle elongation on deltoid muscle performance a computerised study. Clin Biomech. 17(7):499-505, 2002.

[26] D. A. Neumann. Kinesiology of the musculoskeletal system foundations for rehabilitation. St Louis Mosby, pp 13, 2010.

[27] J. H. Weon, O. Y. Kwon, H. S. Cynn, et al. Real-time visual feedback can be used to activate scapular upward rotators in people with scapular winging: an experimental study. J Physiother. 57:101-107, 2011.

[28] N. T. Antony, P. J. Keir. Effects of posture, movement and hand load on shoulder muscle activity. J Electromyogr Kinesiol. 20:191-198, 2010.

[29] P. M. Ludewig, T. M. Cook. Alterations in shoulder kinematics and associated muscle activity in people with symptoms of shoulder impingement. Phys Ther. 80:276-291, 2000.

[30] C. W. MacDonell, P. J. Keir. Interfering effects of the task demands of grip force and mental processing on isometric shoulder strength and muscle activity. Ergonomics. 48:1749-1769, 2005.

[31] D. A. Neumann. Kinesiology of the musculoskeletal system foundations for rehabilitation. St Louis Mosby, pp 48-49, 2010 . 